\title{
A-scope analysis of subsurface radar sounding of lunar mare region
}

\author{
Takao Kobayashi ${ }^{1}$, Hiroshi Oya ${ }^{2}$, and Takayuki Ono ${ }^{1}$ \\ ${ }^{1}$ Tohoku University, 980-8578, Japan \\ ${ }^{2}$ Fukui University of Technology, 910-4272, Japan
}

(Received December 28, 2001; Revised September 13, 2002; Accepted September 29, 2002)

\begin{abstract}
Lunar Radar Sounder (LRS) is a spaceborne HF radar system and is a science mission of Japanese lunar exploration project, SELENE, which is scheduled to be launched in 2005. The primary objective of LRS is to investigate the geologic structure of lunar subsurface from orbit. Computer simulations of LRS observation of lunar mare region have been carried out by utilizing a newly developed simulation code, the Kirchhoff-approximation Sounding Simulation (KiSS) code. The purpose of the simulations is to understand the nature of reflection/refraction of HF wave at the lunar surface as well as at the lunar subsurface boundary, and to confirm that the lunar subsurface structure can be investigated from orbit by means of an HF radar. Gaussian random rough surfaces are employed to represent the surface feature of a lunar mare region. From simulation results, we have found that the power flux of both surface nadir echo and subsurface nadir echo vary little if roughness of either/both surface or/and subsurface boundary interface changes. However, their intensity of surface off-nadir backscattering echo varies following a power law of $\left(k \sigma_{0}\right)^{2}$, where $k$ is the wave number of LRS transmission pulse, and $\sigma_{0}$ is the RMS height of the surface. Thus slight roughness of the surface causes significant increase of the power flux of surface offnadir backscattering echo, which easily masks weak subsurface echoes. These observations have been understood qualitatively by geometrical optics approximation and quantitatively by examining the Stratton's integral formula in an analytic way. Computer simulations have revealed that subsurface echoes are received even if they are completely masked by surface off-nadir backscattering echo. To distinguish those subsurface echoes from strong surface backscattering echo, the data stacking technique has been proved to be effective on reducing surface backscattering echoes due to their random nature.
\end{abstract}

\section{Introduction}

SELENE is Japanese lunar exploration project, which is scheduled to be launched in 2005 . The primary purpose of SELENE project is to obtain scientific data for the study of origin and evolution of the Moon as well as to obtain engineering data for the study of future lunar exploration technology. 14 science missions will be onboard SELENE to conduct measurements and observations of lunar surface, the lunar environment space, and the lunar subsurface space.

Lunar Radar Sounder (LRS) experiment is one of onboard science missions whose primary objective is to investigate lunar subsurface structure. LRS is a spaceborne ground penetrating radar and the apparatus of LRS is an FMCW radar system of HF band. It has been developed as an extension of series of plasma sounders of which technology has been developed through the series of earth orbiting satellites, EXOSB (Oya et al., 1981), EXOS-C (Oya et al., 1985), and EXOSD (Oya et al., 1990), and Mars orbiter PLANET-B (Ono et al., 1998) that is to carry out sounding observation of Martian ionosphere and the surface.

The center frequency of transmission pulse of LRS is $5 \mathrm{MHz}$ and the swept frequency range is $2 \mathrm{MHz}$, which gives the range resolution of $75 \mathrm{~m}$ in vacuum. In completely dry material of lunar subsurface, an electromagnetic wave can

Copy right(c) The Society of Geomagnetism and Earth, Planetary and Space Sciences (SGEPSS); The Seismological Society of Japan; The Volcanological Society of Japan; The Geodetic Society of Japan; The Japanese Society for Planetary Sciences. propagate much deeper than in the subsurface of the earth. In general, in HF frequency range, lower frequency electromagnetic waves can propagate deeper. The frequency of LRS transmission pulse was chosen as a trade-off among allowed instrumental resources, required range resolution and the detection depth. The detail description of LRS hardware is found in Ono and Oya (2000).

LRS detects lunar subsurface echoes which are reflected from subsurface boundary interface where sudden change of dielectric constant of the subsurface material occurs in the direction of depth. The global mapping of observed subsurface echoes are to be used to deduce a lunar subsurface structure model which will provide rich information to reconstruct the evolution history of the Moon (Yamaji et al., 1998).

A similar attempt has been made once in the history of lunar exploration. In 1972, Apollo Lunar Sounder Experiment (ALSE) was conducted in Apollo 17 mission (Phillips et al., 1973), of which primary target was subsurface electrical conductivity structure of the Moon. ALSE was operated in three frequency ranges: $5 \mathrm{MHz}, 15 \mathrm{MHz}$, and 150 MHz. However, ALSE observation time was strictly limited since Apollo 17 was a manned mission. The total amount of recoded $\mathrm{HF}(5 \mathrm{MHz})$ sounder data was of only $31666 \mathrm{sec}-$ ond time (Cooper, 1992), or a little less than 8 hours. Unfortunately Apollo project itself was closed down soon after Apollo 17 mission completed, and, as a result, ALSE data were prevented from being thoroughly analyzed. Neverthe- 
less a portion of ALSE data, mainly of mare region, was analyzed and detection of subsurface echo was confirmed (Phillips et al., 1973). The main problem first to be solved was surface off-nadir echoes (clutters) which were observed in the same range bin as of subsurface echoes. Later, Peeples et al. (1978) analyzed the ALSE data to deduce subsurface structures of Mare Serenitatis and Mare Crisium. Subsurface echoes were manually picked up from ALSE data in their analysis. Data analysis of observation of a highland region had more difficulty in distinguishing subsurface echoes from surface clutters (Cooper et al., 1994).

That ALSE data was recorded in optical films prevented the problem being properly solved. As to LRS, all the data will be processed and recorded in digital format, hence it is expected that obtained data will be analyzed more efficiently. Yet, the data analysis methodology for LRS observation data has not been established. To establish a data analysis methodology for the LRS data in order to distinguish subsurface reflections from surface off-nadir echo, the nature of HF wave propagation should be understood well.

We have developed a computer simulation code, the Kirchhoff-approximation Sounding Simulation (KiSS) code that simulates LRS observation of the Moon with high fidelity so that we investigate the propagation of LRS pulse including both reflection and refraction at the lunar surface and the subsurface boundary. The present study was done on those simulations of LRS observation.

The purpose of the present study is, firstly, to understand the nature of $\mathrm{HF}$ wave propagation through/at the lunar rough surface and of HF wave reflection from a subsurface boundary interface, secondly, to confirm that the lunar subsurface structure can be investigated from orbit by means of an HF radar, and thirdly, to estimate the surface roughness of a lunar mare region by a cross check of the simulation results and the ALSE results.

This paper exclusively treats LRS observations for the case of lunar mare region. LRS observation of a highland region is treated in the companion paper (Kobayashi et al., 2002).

\section{Simulation Code, Model, and Condition 2.1 Simulation code}

The KiSS code has been developed to simulate the end to end sequence of LRS observation, from transmission of a radar pulse to data analysis of the received signal. The core part of KiSS code consists of a subprogram which numerically solves the reflection/refraction problem of LRS pulse at lunar surface and at lunar subsurface boundary interface. The subprogram is designed based on Kirchhoff theory (Beckmann and Spizzichino, 1963). A local reflection/refraction problem on each surface segment is solved in Fresnel's model.

The theory provides the fields of reflected and refracted electromagnetic waves on each side of the media interface, which then are used as the boundary condition of Stratton's integral formula (Stratton, 1941),

$$
\mathbf{E}\left(x^{\prime}, y^{\prime}, z^{\prime}\right)=-\int_{S}\left[\left(\mathbf{n}_{0} \cdot \mathbf{E}_{0}\right) \nabla_{0} G+\left(\mathbf{n}_{0} \times \mathbf{E}_{0}\right)\right.
$$

$$
\left.\times \nabla_{0} G+\left(\mathbf{n}_{0} \times \nabla_{0} \times \mathbf{E}_{0}\right) G\right] d s
$$

to determine the wave field at given distant point (either the observation point or incident point on the subsurface boundary), $\left(x^{\prime}, y^{\prime}, z^{\prime}\right)$. In (1), $\mathbf{E}$ is the electric field vector at the point $\left(x^{\prime}, y^{\prime}, z^{\prime}\right)$. Subscript 0 in the right hand side refers to of the surface. $\mathbf{n}_{0}$ is the unit vector of the local surface normal at incident point $\left(x_{0}, y_{0}, z_{0}\right)$ of the LRS pulse, $\mathbf{E}_{0}$ is the electromagnetic wave field vector of the incident LRS pulse, and $G$ is the Green's function given as

$$
G=\frac{e^{i k r}}{4 \pi r}
$$

where

$$
r=\sqrt{\left(x^{\prime}-x_{0}\right)^{2}+\left(y^{\prime}-y_{0}\right)^{2}+\left(z^{\prime}-z_{0}\right)^{2}}
$$

and $k$ is the wave number vector of the reflected/refracted wave.

Space over lunar surface is, as Ono and Oya (2000) pointed out, filled with either earth's magnetospheric plasma or solar wind plasma that has dispersion effect on electromagnetic wave propagation. However, the density of plasmas is so small that its dispersion effect on the propagation of LRS pulse is negligible. Therefore, the space over the lunar surface is considered as a vacuum in the present simulation.

Multiple scattering is not considered in the present model, which leads to underestimation of depolarization (Ogilvy, 1992). However, its influence on our simulation is anticipated to be little because the depolarization in specular backscattering reflection is very small unless the surface roughness is very large: our primary target, subsurface nadir echo, is a specular backscattering echo and surface with large roughness is not considered in the present simulation.

Shadowing effect is not considered either, which is thought to have little effect on the present simulation as well, because incidence angle of LRS pulse to the mean surface of the Moon is at the most $40^{\circ}$ while the slope of the lunar surface topography is much smaller than $40^{\circ}$ in most part of the lunar mare surface.

\subsection{Simulation model}

The electric field of LRS pulse incident upon lunar surface is given as the radiation field of a small electric dipole antenna. The approximation should be valid because the spatial distance between the lunar surface and LRS antenna (100 $\mathrm{km})$ is very large comparing to the wavelength of LRS pulse $(60 \mathrm{~m})$

The mean surface of the Moon is considered as a flat plane because the global curvature would not play an essential role in the present study.

The Moon is modeled to have two layers (Fig. 1). The upper boundary of the upper layer is the interface to vacuum and is given a surface topography. The lower boundary of the upper layer is the interface to the lower layer, which is also given a topography independent of the surface topography. The upper layer is as thick as $1000 \mathrm{~m}$. Each layer is filled with uniform material of which dielectric constant is $\varepsilon_{1}=$ $4.0+i 0.05$ for the upper layer and $\varepsilon_{2}=8.0+i 0.05$ for the lower layer. These complex values of dielectric constants are 


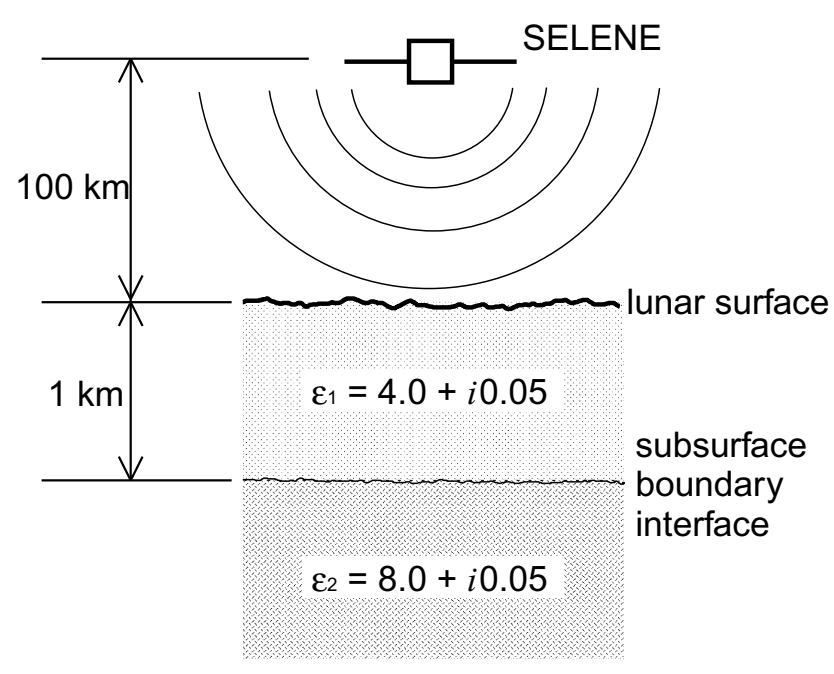

Fig. 1. A schematic sketch of the simulation model. The surface and the subsurface boundary are given independent Gaussian random rough surface features.

commonly found in measured data of Apollo lunar samples (Olhoeft and Strangway, 1975; Heiken et al., 1991).

The surface extension is set as a square of $200 \mathrm{~km} \times 200$ $\mathrm{km}$ so that no reflected/diffracted wave from the simulation space boundary is received while the receiving window of LRS is opened. The extension of subsurface boundary is set as a square of $20 \mathrm{~km} \times 20 \mathrm{~km}$ in order to carry out the simulation in a practical time period. Calculation of subsurface propagation is a heavily resource consuming job because Stratton's integral should be carried out to obtain the electric field of incident wave upon every lattice point on the boundary surface. The lattice interval is chosen $10 \mathrm{~m}$, therefore, the extension of the subsurface boundary has $4 \times 10^{6}$ lattice points.

\subsection{Mare surface model and validity condition}

The surface of a lunar mare region is regarded as a random rough surface because it has no distinct terrain feature except scattered impact craters. Among various random rough surface of natural origin, the most widely observed is Gaussian random rough surface (Thomas, 1999). Thus we employ Gaussian random rough surface to model the surface topography of a lunar mare region.

The statistical property of a Gaussian random rough surface is determined by the correlation length and the RMS height: the correlation length determines the horizontal scale of the surface roughness and the RMS height determines the vertical scale of the roughness. A Gaussian random rough surface is described by the power spectrum of the surface roughness which takes the form of

$$
P\left(k_{x}, k_{y}\right)=\frac{\sigma^{2}}{4 \pi \lambda_{x} \lambda_{y}} \exp \left(-\frac{k_{x}^{2} \lambda_{x}^{2}+k_{y}^{2} \lambda_{y}^{2}}{4}\right)
$$

where $\lambda_{x}, \lambda_{y}, \sigma$, are the correlation length in $x$ direction, the correlation length in $y$ direction, RMS height of the surface roughness, respectively, and, $k_{x}, k_{y}$ are wavenumber of roughness in $x$ direction and $y$ direction, respectively.

In our simulation, we assume the same correlation length in both $x$ and $y$ directions as

$$
\lambda_{x}=\lambda_{y}=\lambda_{0}
$$

Therefore the roughness of Gaussian random rough surface in our simulation is of isoscale in horizontal direction.

One should be careful in choosing the values of surface parameters in order to keep the validity condition required by Kirchhoff theory. So far, there has been established no such formula that gives the validity limit of the theory. However, it is widely accepted that the theory is valid under the condition

$$
k r_{c} \cos \theta_{\text {in }} \gg 1
$$

where $k$ is the wavenumber of the wave of concern (LRS transmission pulse), $r_{c}$ is the radius of curvature of the surface, and $\theta_{i n}$ is the incident angle of the wave onto a point on the surface (Ogilvy, 1992). As to a Gaussian random rough surface, the mean radius of curvature of the surface is given as

$$
r_{c}=\frac{\lambda_{0}^{2}}{2 \sqrt{3} \sigma_{0}}\left(1+\frac{2 \sigma_{0}^{2}}{\lambda_{0}^{2}}\right)^{3 / 2}
$$

where $\sigma_{0}$ is the RMS height of the surface with respect to the mean surface, $\lambda_{0}$ is the correlation length of the surface (Ogilvy, 1992). In our simulation model, $\lambda_{0}$ is much larger than $\sigma_{0}$, therefore Eq. (7) becomes

$$
r_{c} \sim 0.3 \frac{\lambda_{0}^{2}}{\sigma_{0}}
$$

Further, $\cos \theta_{\text {in }}$ is approximated as unity because we do not treat large incident angle of LRS pulse onto the lunar surface. Thus Eq. (6) is modified as

$$
0.3 \frac{\left(k \lambda_{0}\right)^{2}}{k \sigma_{0}} \gg 1 \text {. }
$$

The simulation study must be carried out under the condition of (9).

Since we have no hypsometric information of lunar mare region in fine scale, infinite number of combinations of surface roughness parameters, i.e. the correlation length and the RMS height, ought to be investigated. Considering, however, that an alternative property of a Gaussian random rough surface, RMS gradient, is often referred to as the representative statistical property of a rough surface in radar study of planetary surface, we may fix either of correlation length or RMS height at any value and change the rest in our simulation study rather than investigate all the possible combinations of surface roughness parameters, because RMS gradient of a Gaussian random rough surface, $\theta_{R M S}$, is expressed as

$$
\tan \theta_{R M S}=\sqrt{2} \frac{\sigma_{0}}{\lambda_{0}}
$$

and has common value to different combinations of surface roughness parameters so far as the ratio of parameters is common.

A nondimensional number $k \sigma_{0}$ gives an index of the surface roughness relative to the wave length of transmission pulse. We investigate the effect of the roughness of lunar 
Table 1. LRS parameter.

\begin{tabular}{|c|c|c|}
\hline SELENE altitude & & $100 \mathrm{~km}$ above the mean surface \\
\hline SELENE velocity & & $1.6 \mathrm{~km} / \mathrm{sec}$, parallel to the mean surface \\
\hline Doppler shift & & ignored \\
\hline Power & & $P=800 \mathrm{~W}$ \\
\hline Antenna impedance & & $Z=50 \Omega($ no frequency dependence considered) \\
\hline Antenna length & & $L=30 \mathrm{~m}$ \\
\hline Frequency & & $f=4 \nearrow 6 \mathrm{MHz}$ (linear sweep) \\
\hline Pulse length & & $T=200 \mu \mathrm{sec}$ \\
\hline $\begin{array}{l}\text { Pulse repetition } \\
\text { Pulse envelope } \\
\text { Weight function }\end{array}$ & & $\begin{array}{lr}f_{\text {rep }}=20 \mathrm{~Hz} & \begin{array}{lr}\frac{1}{2}\left\{1-\cos \left(10 \frac{t}{T}\right) \pi\right\} & \left(0 \leq t<\frac{1}{10} T\right) \\
1 & \left(\frac{1}{10} T \leq t \leq \frac{9}{10} T\right) \\
\frac{1}{2}\left\{1+\cos \left(10 \frac{t}{T}-9\right) \pi\right\} & \left(\frac{9}{10} T<t \leq T\right)\end{array}\end{array}$ \\
\hline
\end{tabular}

surface on LRS observation in the range of validity condition (9) at a fixed correlation length $\lambda_{0}=60 \mathrm{~m}$. And thereafter we analyse the simulation result, in comparison with ALSE observation data in terms of surface RMS gradient. The wave number of LRS transmission pulse, $k$, is $2 \pi / 60$ $\left[\mathrm{m}^{-1}\right]$. Therefore, the simulation is valid under the condition

$$
k \sigma_{0} \ll 12 .
$$

\subsection{Simulation condition}

General conditions of simulation in the present study are based on the planned SELENE operation parameters and LRS hardware parameters. They are listed in the table.

\section{Single Pulse Observation}

\subsection{Surface reflection}

We first examined the effect of surface roughness on single pulse observations. The roughness of the subsurface boundary interface is assumed to be 0 , i.e. $k \sigma_{1}=0$.

Figure 2 shows the results from the simulation of LRS observations in the form of A-scope (a plot of signal amplitude versus range). The received signal amplitude is presented as the power flux at the SELENE spacecraft and the range is offset for $100 \mathrm{~km}$ so that the zero range coincides with the nadir point on the mean surface.

From the top panel to the bottom in Fig. 2, the surface roughness parameter, $k \sigma_{0}$, varies from 0 to $0.03,0.1$, and 0.3 as respectively indicated at the right of each panel in the figure.

Those echoes which appeared in the A-scope displays may be categorized in three components:

(1) surface nadir echo,

(2) surface off-nadir backscattering echo, and

(3) subsurface nadir echo.

The surface nadir echo is the most intense signal that is found at the zero range. The surface off-nadir backscattering

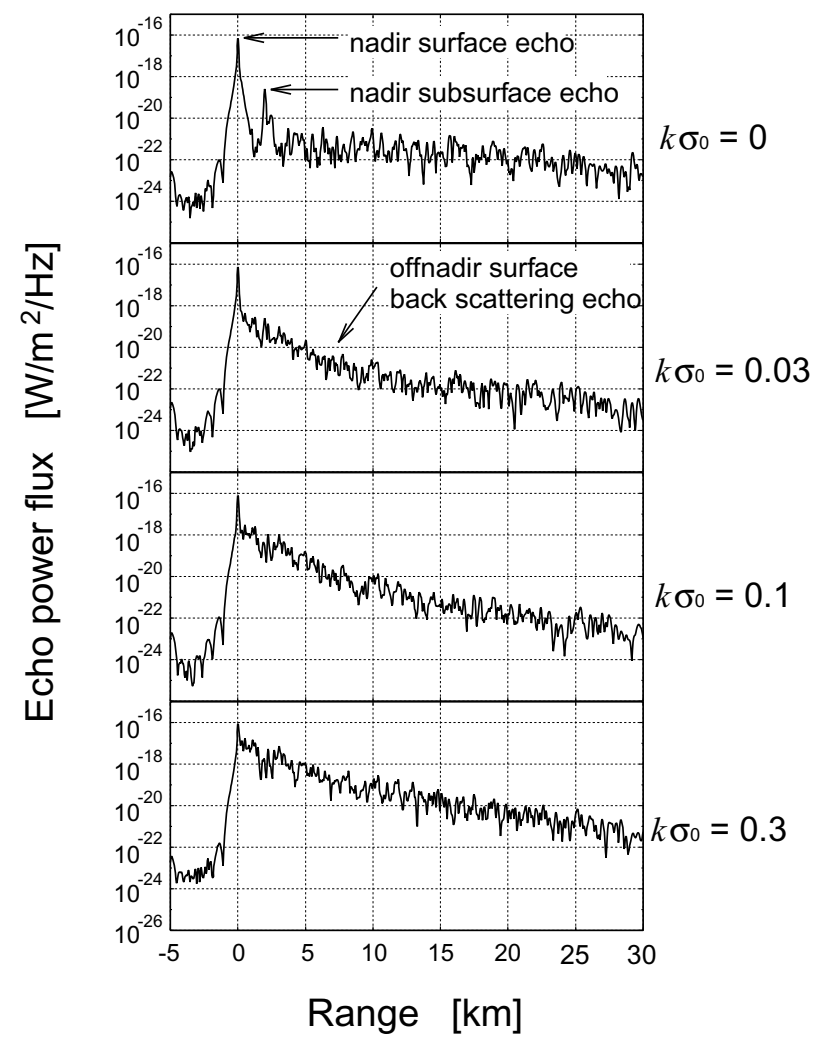

Fig. 2. A-scope displays of single pulse observations of LRS. Range is offset for $100 \mathrm{~km}$ so that the zero range coincides with the nadir point on the mean surface. The surface roughness parameter, $k \sigma_{0}$, increases from the top panel to the bottom as indicated at the right of each panel.

echo is found in any range larger than the nadir range, except in the case of $k \sigma=0$. In this case, the signals that seem to be the surface off-nadir backscattering echo is discretization noise which should be attributed to the finite lattice dimension of the simulated lunar surface. The subsurface nadir echo is found at the apparent range of $2 \mathrm{~km}$ as a small signal 
peak, which is about $25 \mathrm{~dB}$ less in power than the surface nadir echo.

It is apparent from Fig. 2 that the surface nadir echo intensity is insensitive to the change of the surface roughness while the surface off-nadir backscattering echo is very sensitive to it: only slight roughness of the surface can cause significant increase in the intensity of surface off-nadir backscattering echo so that the subsurface nadir echo is lost among surface echoes in an A-scope display.

These observations are quantitatively verified by Fig. 3 in which both the surface nadir echo (open circle) and the surface off-nadir backscattering echo (closed circle) are plotted as functions of the surface roughness parameter, $k \sigma_{0}$.

The echo power in the Fig. 3 has been normalized by the surface nadir echo intensity of the case of $k \sigma_{0}=0$. And we have defined the power of the surface off-nadir backscattering echo $P_{\text {off-nadir }}$ as the mean power of surface off-nadir backscattering echo in the range from $1 \mathrm{~km}$ to $5 \mathrm{~km}$ as follows

$$
P_{\text {off-nadir }}=\frac{1}{P_{0}}\left[\prod_{n=N_{1}}^{N_{5}} P_{n}\right]^{1 /\left(N_{5}-N_{1}+1\right)}
$$

where $P_{n}$ is the power of the echo that is received in the $n$th range bin, $N_{1}$ is the number of the range bin whose range is $1 \mathrm{~km}, N_{5}$ is the number of the range bin whose range is $5 \mathrm{~km}$, and $P_{0}$ is the normalization factor, the power of the surface nadir echo of the case of $k \sigma_{0}=0$. The error bars of in Fig. 3 depict the maxima and minima of observed power flux of off-nadir backscattering echoes in the definition range of off-nadir echo.

Superimposed lines indicate power laws: the null power law for the case of surface nadir echo and the square power law of $k \sigma_{0}$ for the case of surface off-nadir backscattering echo. We have now learned these two categories of surface echoes show different responses to the change of surface roughness in contrast with each other. The nature of these

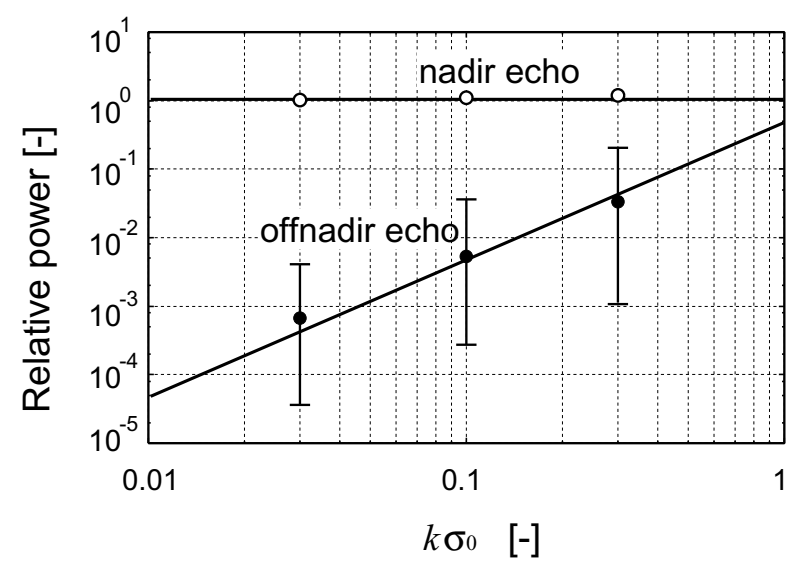

Fig. 3. Power flux variation of surface nadir echo and surface off-nadir backscattering echo of single pulse observation as functions of the surface roughness parameter, $k \sigma_{0}$. Power flux is normalize by the power flux of the surface nadir echo of single pulse observation of the case of $k \sigma_{0}=0$. Superimposed are lines of two power laws, $\left(k \sigma_{0}\right)^{0}$ and $\left(k \sigma_{0}\right)^{2}$. The error bars of off-nadir backscattering echo depict the maxima and minima of observed power flux in the definition range of off-nadir echo. property is understood qualitatively by geometrical optics approximation and quantitatively by examining Stratton's integral formula analytically.

(a) Geometrical optics consideration Figure 4 schematically shows LRS observation in terms of geometrical optics approximation where solid lines represent ray paths of incident waves of LRS transmission pulse upon the lunar surface and dashed lines represent ray paths of reflected waves.

The surface is divided into two regions. One is the nadir range region, of which any target is detected in the range bin to which the nadir surface point (suborbital point of the SELENE) belongs. Rest of the surface belongs to the offnadir range region.

As Fig. 4 shows, for the case of $k \sigma_{0}=0$, the wave energy that incidents upon the off-nadir region is never reflected back toward the LRS receiving antenna: all the energy is reflected off the LRS receiving antenna. The situation as to the nadir range region is different; while some portion of incident wave energy is reflected off the LRS antenna, certain amount of incident wave energy is reflected back toward LRS antenna, which makes the nadir echo peak in an A-scope.

However, in the case of $k \sigma_{0}>0$, some portion of wave energy that incidents upon off-nadir range region is reflected back toward the LRS receiving antenna because, at some points, the specular reflection coincides with the backscattering direction due to the local gradient of the surface which is no longer 0 .

As to the nadir range region in the case of $k \sigma_{0}>0$, some portion of such incident wave energy that would be reflected back toward the LRS receiving antenna if $k \sigma_{0}$ is 0 , is not reflected back in the same direction due to the local surface gradient. However the opposite occurs to other portion of such incident wave energy that would not be reflected back toward the LRS receiving antenna if $k \sigma_{0}$ is 0 but is reflected back toward the LRS antenna due to the local surface gradient. These changes of reflection direction take place randomly since what is responsible for these changes is the distribution of surface gradient which is a random phenomenon. And, so far as the surface roughness is small, the discrepancy between these two portions of energy is expected to be small. As a result, the total amount of the nadir echo intensity changes little in the simulations.

According to the geometrical optics approximation, no energy should be reflected back toward the LRS antenna if the surface is a smooth plane, $k \sigma_{0}=0$. But the A-scope of the top panel in Fig. 2, the case of $k \sigma_{0}=0$, shows that weak surface clutter echoes are certainly observed in the off-nadir range region. As mentioned above, this component of surface echo is attributed to numerical noises due to finite discretization of the surface topography. Therefore one should be careful about that small amount of numerical noise is contained in the simulation results when treating very weak signals.

(b) Analytic consideration As is observed in Fig. 3, the nadir range echo has no dependence on the surface roughness $k \sigma_{0}$ while the off-nadir range echo has apparently strong dependence on the surface roughness through an empirical 

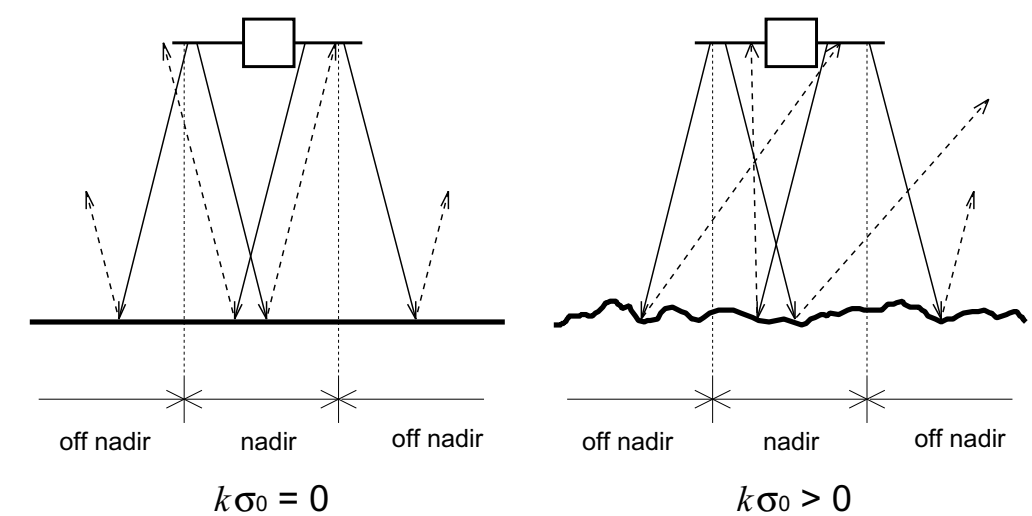

Fig. 4. Geometrical optics approximation of LRS observation. Solid lines represent the ray paths of incident LRS pulses onto the lunar surface and dashed lines represent the ray paths of waves reflected from the surface.

power law of

$$
P_{\text {off-nadir }} \propto\left(k \sigma_{0}\right)^{2} .
$$

Considering that all the received surface reflection echoes are backscattering echoes (because the LRS observation is a monostatic radar observation) the relation of (13) can be derived analytically by applying backscattering condition

$$
\mathbf{k}_{s c}=-\mathbf{k}_{\text {in }}
$$

to (1), where $\mathbf{k}_{\text {in }}$ is incident wave number vector and $\mathbf{k}_{s c}$ is reflected wave number vector. Applying (14) to (1), after some manipulation of integrand, one obtains

$$
\mathbf{E}^{s c}(\mathbf{r})=-i k \frac{e^{i k r}}{2 \pi r} \int_{S_{0}}\left\{\mathbf{n}_{0}\left(\mathbf{r}_{0}\right) \cdot \mathbf{k}_{i n}\right\} \mathbf{E}_{0}\left(\mathbf{r}_{0}\right) e^{i \mathbf{k}_{i n} \cdot \mathbf{r}_{0}} d S_{0}
$$

where $\mathbf{E}^{s c}(\mathbf{r})$ is the electric field vector of surface reflection wave at the observation point, $\mathbf{r}$. Taking Poynting vector of (15) on the reflection surface yields

$$
\mathbf{P}^{s c}(\mathbf{r})=k^{2} \frac{e^{i 2 k r}}{4 \pi^{2} r^{2}} \sqrt{\frac{\mu}{\varepsilon}} \int_{S_{0}}\left\{\mathbf{n}_{0}\left(\mathbf{r}_{0}\right) \cdot \mathbf{k}_{i n}\right\}^{2} E_{0}^{2}\left(\mathbf{k}_{i n}\right) e^{i \mathbf{k}_{i n} \cdot \mathbf{r}_{0}} d S_{0}
$$

where $\mathbf{P}^{s c}(\mathbf{r})$ is the Poynting vector of reflection wave at the observation point.

Now we consider the surface feature. For the case of $k \sigma_{0}=0$, surface normal vector is the unit vector of $z$ direction, $(0,0,1)$, at any point on the surface. Thus, in this case, (16) leads to

$$
\mathbf{P}_{k \sigma=0}^{s c}(\mathbf{r})=k^{2} \frac{e^{i 2 k r}}{4 \pi^{2} r^{2}} \sqrt{\frac{\mu}{\varepsilon}} \int_{S_{0}} k_{z}^{2} E_{0}^{2} \mathbf{k}_{i n} e^{i \mathbf{k}_{i n} \cdot \mathbf{r}_{0}} d S_{0}
$$

which is the Poynting vector of the reflection wave from $k \sigma_{0}=0$ surface.

Next, we introduce surface roughness. Surface normal vector of a continuously smooth rough surface is expressed as

$$
\mathbf{n}_{0}\left(\mathbf{r}_{0}\right)=\left(-\frac{\partial h}{\partial x},-\frac{\partial h}{\partial y}, 1\right)
$$

If the surface gradient is very small, it would be allowed that surface normal unit vector is approximated by (18). This approximation modify (16) as

$$
\begin{aligned}
\mathbf{P}_{k \sigma>0}^{s c}(\mathbf{r})= & k^{2} \frac{e^{i 2 k r}}{4 \pi^{2} r^{2}} \sqrt{\frac{\mu}{\varepsilon}} \int_{S_{0}} k_{z}^{2} E_{0}^{2} \mathbf{k}_{i n} e^{i \mathbf{k}_{i n} \cdot \mathbf{r}_{0}} d S_{0} \\
& +k^{2} \frac{e^{i 2 k r}}{4 \pi^{2} r^{2}} \sqrt{\frac{\mu}{\varepsilon}} \int_{S_{0}} \Delta E_{0}^{2} \mathbf{k}_{i n} e^{i \mathbf{k}_{i n} \cdot \mathbf{r}_{0}} d S_{0}
\end{aligned}
$$

where

$$
\begin{aligned}
\Delta= & \left(\frac{\partial h}{\partial x}\right)^{2} k_{x}^{2}+\left(\frac{\partial h}{\partial y}\right)^{2} k_{y}^{2} \\
& +2\left(\frac{\partial h}{\partial x}\right)\left(\frac{\partial h}{\partial y}\right) k_{x} k_{y} \\
& -2\left(\frac{\partial h}{\partial y}\right) k_{y} k_{z}-2\left(\frac{\partial h}{\partial x}\right) k_{z} k_{x}
\end{aligned}
$$

The first term in right hand side of (19) is identical to (17), which represents the nadir echo. Thus the second term represents the off-nadir echoes. Since (17) is free of $\sigma_{0}$, the nadir echo is independent of surface roughness $k \sigma_{0}$ for small $\sigma_{0}$ value.

Let us remind that height distribution of the surface follows Gaussian statistics. Under this assumption, a distribution function of the surface gradient is given as

$$
\begin{aligned}
& P\left(\frac{\partial h}{\partial x}, \frac{\partial h}{\partial x}\right) \\
& \quad=\frac{\lambda_{0}^{2}}{4 \pi \sigma_{0}^{2}} \exp \left[-\frac{\lambda_{0}^{2}}{4 \sigma_{0}^{2}}\left\{\left(\frac{\partial h}{\partial x}\right)^{2}+\left(\frac{\partial h}{\partial y}\right)^{2}\right\}\right]
\end{aligned}
$$

Using (21), the expectation of the second term in right hand side of (19), $\left\langle P_{\text {off-nadir }}\right\rangle$, is obtained as

$$
\begin{aligned}
\left\langle P_{\text {off-nadir }}\right\rangle= & \left(k \sigma_{0}\right)^{2} \frac{1}{\lambda_{0}^{2}} \frac{e^{i 2 k r}}{2 \pi^{2} r^{2}} \sqrt{\frac{\mu}{\varepsilon}} \\
& \times \int_{S_{0}}\left(k_{x}^{2}+k_{y}^{2}\right) E_{0}^{2} \mathbf{k}_{i n} e^{i \mathbf{k}_{i n} \cdot \mathbf{r}_{0}} d S_{0} .
\end{aligned}
$$

Note that the coefficient $\left(k \sigma_{0}\right)^{2}$ is separated out of integrand. This term makes surface off-nadir echo intensity be proportional to the square of $k \sigma_{0}$ as has been shown in (13) (and Fig. 3). 


\subsection{Subsurface reflection}

Even slight roughness of the surface (small value of $k \sigma_{0}$ ) makes the intensity of surface off-nadir backscattering echo increase large enough to mask the subsurface nadir echo (Fig. 2) in an A-scope. A question arises whether subsurface echoes still return to LRS receiver when they are completely masked by intense surface off-nadir backscattering echoes. We have carried out simulations in which subsurface reflection is separated from surface reflection in the process of calculation so that we can estimate the effect of surface roughness (and roughness of subsurface boundary interface) exclusively on the subsurface reflections.

The simulation includes penetration through the surface and reflection at the subsurface boundary interface under several different conditions of $k \sigma_{0}$ (surface roughness) and $k \sigma_{1}$ (roughness of subsurface interface). The results are shown in Fig. 5 in the form of A-scope displays. In Fig. 5, only subsurface echo signals are displayed in each panel. Since the extent of subsurface boundary interface is limited to a square area of $20 \mathrm{~km} \times 20 \mathrm{~km}$, the duration range of subsurface echo is significantly limited as has been shown in Fig. 5. Our interest is in the largest intensity of subsurface echo presented in the figure.

In Fig. 5, sixteen A-scopes are juxtaposed. From top to down in each column, the value of $k \sigma_{0}$ increases in the same order as Fig. 2, i.e. 0, 0.03, 0.1, and 0.3. From left to right in each row, the value of $k \sigma_{1}$ increases in the same manner.

All the subsurface nadir echoes appear in Fig. 5 at the apparent range of $2 \mathrm{~km}$ in A-scopes because the subsurface interface is located at the depth of $1000 \mathrm{~m}$ below the mean

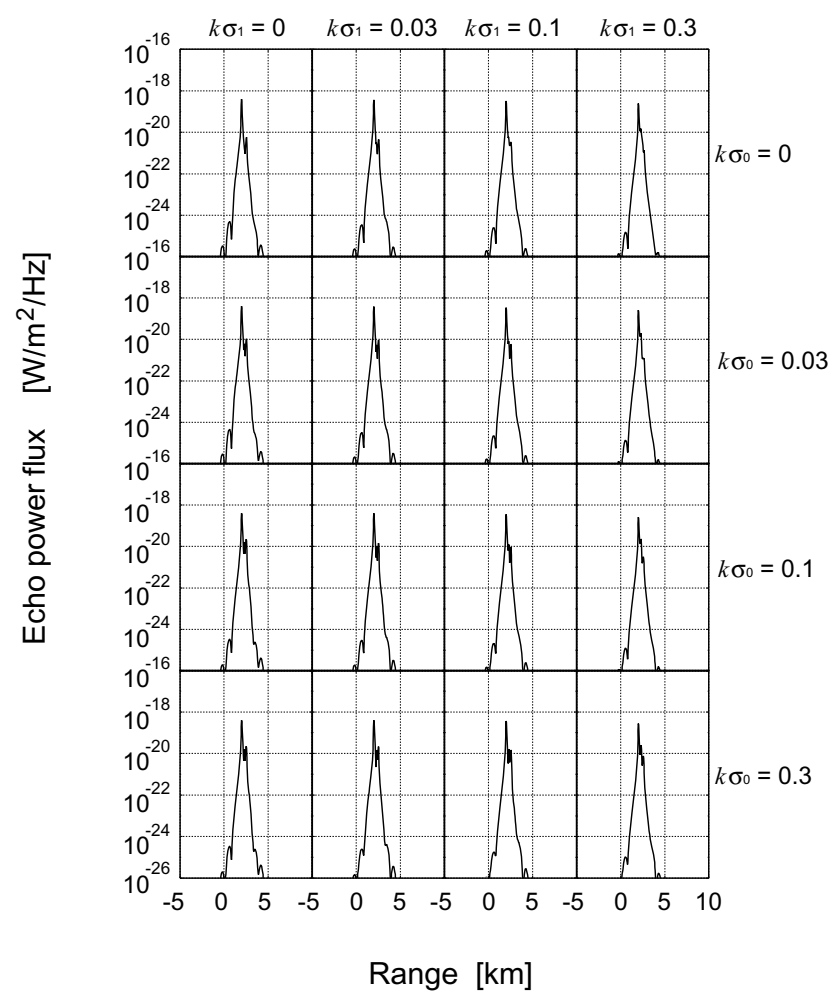

Fig. 5. A-scope displays of subsurface echoes as functions of $k \sigma_{0}$ and $k \sigma_{1}$. Only subsurface echoes are displayed. $k \sigma_{0}$ increases from the top panel to the down as in Fig. 2, $k \sigma_{1}$ increases from the left panel to the right as indicated above the panels. surface while the dielectric constant is set $4.0+i 0.05$ for the lunar subsurface material. Figure 5 shows little variation in subsurface nadir echo despite the change of both surface roughness, $k \sigma_{0}$, and the roughness of the subsurface boundary interface, $k \sigma_{1}$, as has been the case with surface nadir echo. The reason for this unique property of subsurface nadir echo can be explained in the same manner as for the case of surface nadir echo. Thus, so far as the depth of subsurface interface changes none or a very little compared to the wave length of LRS pulse during the observation period (along the course of orbit motion of the spacecraft), the subsurface nadir echoes can be regarded not only as coherent signals as the surface nadir echo is, but also as stable signals whose intensity changes little, independent of either surface roughness, $k \sigma_{0}$, or subsurface boundary roughness, $k \sigma_{1}$, though they are very weak comparing to surface off-nadir echoes.

The simulation results of Fig. 5 provide us with the answer to our question: a subsurface echo certainly returns to the LRS receiver though it is hardly distinguished from intense surface off-nadir backscattering echoes in an A-scope display as is seen in Fig. 3.

Considering that surface off-nadir backscattering echoes have the property of incoherent signals of which phase varies in random manner from one observation to another, it might be possible to discriminate subsurface echo. Those incoherent signals of surface off-nadir backscattering echoes cancel out each other by stacking observation data in waveform, or in other words, by taking an average of observations. And, if the depth of the subsurface boundary interface does not change rapidly with respect to the move of the spacecraft, the subsurface echo, a coherent signal that is stable independent of either $k \sigma_{0}$ or $k \sigma_{1}$, would change little its intensity through this averaging process while surface off-nadir echoes reduces their intensities, and would be detected.

\section{Effect of Data Stacking}

Let $R_{n}(t)$ be the complex amplitude of a range bin data of the $n$th observation. $R_{n}(t)$ is the ensemble of surface offnadir backscattering echo, $A_{\text {sur }}$, which is strong but incoherent in terms of the observation point, and subsurface echo, $A_{\text {sub }}$, which is weak but coherent in terms of the observation point. Both $A_{s u r}$ and $A_{s u b}$ are complex amplitude. Taking into account of the nature of coherent signal and incoherent signal, we may express the square of mean value of $R_{n}(t)$ as

$$
\left|\frac{1}{N} \sum_{n}^{N} R_{n}\right|^{2} \approx \frac{1}{N}\left\langle\left|A_{\text {sur }}\right|\right\rangle^{2}+\left\langle\left|A_{\text {sub }}\right|\right\rangle^{2}
$$

if $N$ is large enough. Therefore, the mean intensity of the surface off-nadir backscattering echo would be reduced, with respect to the intensity of the mean subsurface nadir echo, inversely proportional to the data stacking number $N$. And, eventually, the subsurface echo would be detectable even if it is too weak to be discerned in the A-scope display of a single pulse observation.

We have examined the effect of data stacking on detecting subsurface echo. 


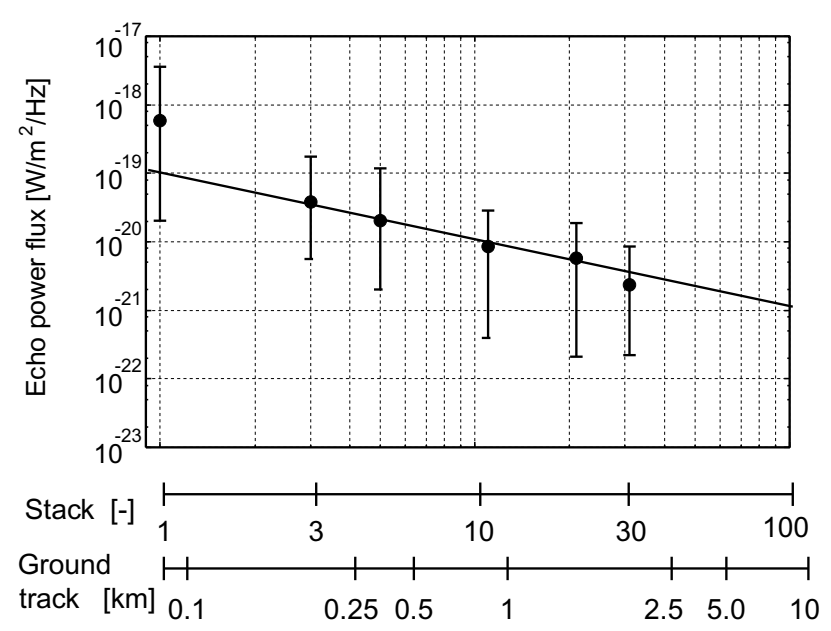

Fig. 6. Effect of data stacking technique on reducing surface off-nadir echo intensity. Surface off-nadir echo intensity of a surface whose roughness is $k \sigma_{0}=0.3$ is plotted as a function of data stack number. The error bars of off-nadir backscattering echo depict the maxima and minima of observed power flux in the definition range of off-nadir echo. The second horizontal axis refers to the ground track of SELENE space craft orbit.

\subsection{Reduction of surface off-nadir backscattering echo intensity}

Simulation of LRS observations over a random rough surface has been carried out in order to demonstrate the effect of data stacking on reducing the intensity of surface off-nadir backscattering echoes. The surface roughness is assumed to be $k \sigma_{0}=0.3$. The total of 31 consecutive LRS observations along an orbit have been simulated. Subsurface reflection is not treated in the simulation.

We examine the behavior of the intensity of surface offnadir backscattering echoes as a function of data stacking number. The intensity is defined as has been in the previous section as (12), i.e. the mean intensity of those echoes which received in the range from $1 \mathrm{~km}$ to $5 \mathrm{~km}$.

Figure 6 presents the results. The intensity of the surface off-nadir backscattering echo is plotted as a function of the number of waveform data stacked, with the line indicating the power law of $N^{-1}$. The definition of error bars is the same as that of Fig. 3. The second horizontal axis in the figure refers to the move of the SELENE spacecraft along the ground track during the observation of which data are stacked. As we have expected, the surface off-nadir backscattering echo reduces its intensity inversely proportional to the data stacking number $N$. The result implies that data stacking technique is an effective mean to reduce surface off-nadir echo intensity in order to detect a weak but coherent subsurface echo.

\subsection{Discerning the subsurface echo}

We have carried out the simulation of 21 consecutive LRS observations in a mare region, in which the surface topography is represented by a Gaussian random rough surface whose roughness is $k \sigma_{0}=0.1$. The subsurface boundary interface is represented by another Gaussian random rough surface whose roughness is also $k \sigma_{1}=0.1$.

The average of these 21 observations has been taken to produce an averaged A-scope data. The result is presented in the form of an A-scope display in Fig. 7 with the A-scope
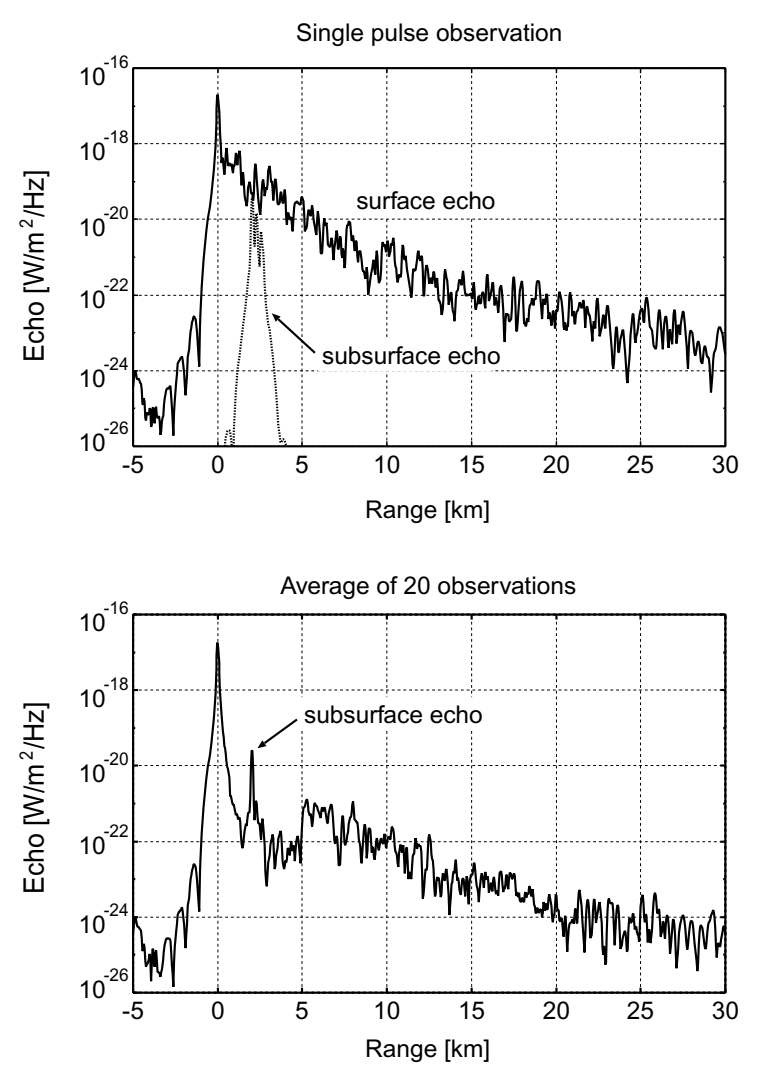

Fig. 7. Effect of data stacking. Upper panel shows an A-scope display of single pulse observation. Roughness of surface is set $k \sigma_{0}=0.1$ and of subsurface interface is set $k \sigma_{1}=0.1$. Solid line is the plot of observed data which contains both surface echo and subsurface echo. Dashed line is the subsurface echo. Note that subsurface echo is completely masked by the surface off-nadir echo. Lower panel shows the A-scope display of 21 data stacking. The effect of data stacking on reducing the surface off-nadir echo is apparent. The subsurface echo appeared at the apparent range of $2 \mathrm{~km}$.

display of a single pulse observation.

The upper panel of Fig. 7 shows the A-scope display of the single pulse observation. In the display, the subsurface echo is plotted separately as the dotted line. It is apparent that the subsurface echo is not discerned from surface off-nadir echoes because it is completely masked.

The lower panel of Fig. 7 shows the averaged A-scope display that is produced from the averaged waveform data of 21 consecutive observations. The data stacking process has reduced the intensity of surface off-nadir echoes for more than $20 \mathrm{~dB}$ and, as a result, the subsurface echo has appeared over the reduced surface off-nadir echoes at the apparent range of $2 \mathrm{~km}$.

From these results above, it is concluded that, in LRS observation of mare region, the data stacking process is effective in detection of subsurface echo even if it is completely masked by surface off-nadir backscattering echoes in an Ascope display of a single pulse observation.

\section{Validity of the Simulation}

Lastly, we verify the validity of the LRS simulation results by comparing with published results of ALSE.

All the observation data of ALSE was recorded in optical films and the recorded data was further processed by optical 
techniques. A small portion of data (2 MB) was transformed into digital format for the purpose of a test. As mentioned above, published papers on ALSE result have not been many. In fact those data which can be used as the reference for our verification are only two A-scope displays in Phillips et al. (1973). One is the A-scope display of a single pulse observation, figure 22-14 of Phillips et al. (1973), and another is an A-scope display of stack-averaged data, figure 22-15 of Phillips et al. (1973).

First we verify the simulation result of single pulse observation. Figure 8 shows an A-scope display in which three Ascope data are plotted, namely, the A-scope data of a single pulse observation of ALSE (thick solid line) with the simulation results of single pulse observations of LRS for the cases of $k \sigma_{0}=0.1$ (thin solid line) and $k \sigma_{0}=0.3$ (dotted line). ALSE data have been read from figure 22-14 in Phillips et al. (1973). Echo power of LRS simulation results is normalized by the surface nadir echo in each case in accordance with ALSE results. Allowing some fluctuation of echo power, the ALSE result can be well fitted by the simulation results of these two cases. Thus, concerning to this particular observation of ALSE, the surface of the mare region (Mare Serenitatis) may be represented by a Gaussian random rough surface whose roughness is $k \sigma_{0}=0.1 \sim 0.3$.

The second verification is for the data stacking technique. Figure 22-15 of Phillips et al. (1973) showed an A-scope display of 300 observation stacked data of Mare Serenitatis. The stacked data were obtained during the flight along the orbit for $15 \mathrm{~km}$. As we have seen in our simulation results, the ALSE 300 stacked data also showed a significant reduction of surface off-nadir echo intensity: the relative intensity of the surface off-nadir echo $(1 \mathrm{~km}<$ range $<5 \mathrm{~km})$ to the surface nadir echo read $-47 \mathrm{~dB}$ (Phillips et al., 1973). Our simulation shows that the relative power of surface off-nadir echo to the surface nadir echo in a single pulse observation is $-23 \mathrm{~dB}$ for the case of $k \sigma_{0}=0.1$ and $-14 \mathrm{~dB}$ for the case of $k \sigma_{0}=0.3$ (Fig. 3). On the other hand, our simulation results of data stacking (Fig. 6) implies that stacking all the data obtained during a $15 \mathrm{~km}$ flight would reduce the intensity of surface off-nadir echoes for $23 \mathrm{~dB}$. Thus, we expect that the relative intensity of surface off-nadir echo, after stacking all

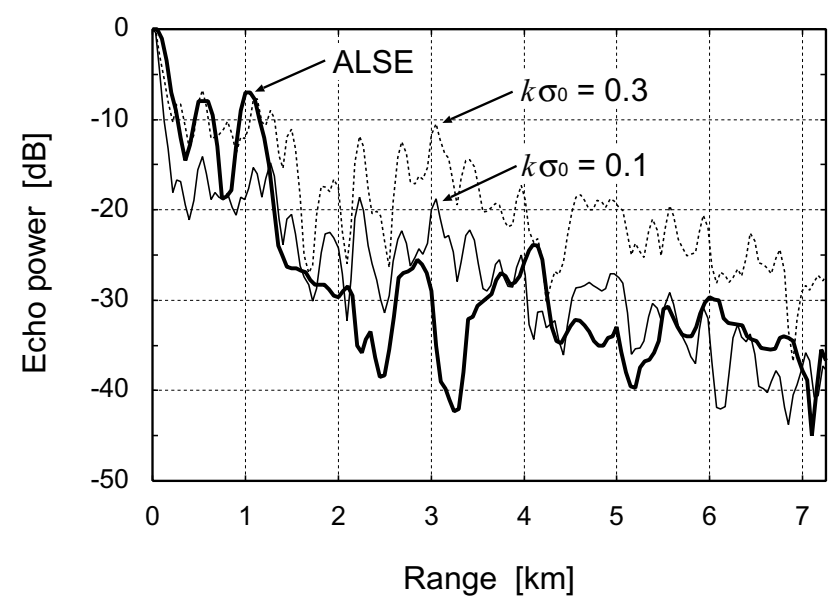

Fig. 8. Comparison of LRS simulation and ALSE observation result (Phillips et al., 1973) in an A-scope display. the data of $15 \mathrm{~km}$ flight, would be $-46 \mathrm{~dB}$ for the case of $k \sigma_{0}=0.1$, which agrees to the ALSE result. In the case of $k \sigma_{0}=0.3$, it would be $-37 \mathrm{~dB}$.

From these verifications, we can conclude that our simulation is verified by the ALSE observation data and that the surface of a lunar mare region (Mare Serenitatis) may be represented by a Gaussian random rough surface whose surface roughness parameters are $k \sigma_{0} \simeq 0.1$ with $\lambda_{0}=60 \mathrm{~m}$.

In this case the RMS gradient of the surface, $\theta_{R M S}=$ $\tan ^{-1}\left(\sqrt{2} \sigma_{0} / \lambda_{0}\right)$, is obtained as

$$
\theta_{R M S} \simeq 1.3^{\circ} \text {. }
$$

The value is smaller than values obtained by some observations such as Simpson (1976) who made ground based radar observations of a lunar mare region, and Simpson and Tyler (1982) who made bistatic observations involving a spaceborne radar. Both of them obtained $4^{\circ}$ as the mean slope of a lunar mare surface. Tyler and Howard (1973) obtained the value that ranges from $2^{\circ}$ to $4^{\circ}$ by bistatic radar investigations in Apollo 14 and 15 missions.

The discrepancy of our result and previous works might be expected considering that those authors had used much shorter wavelength (i.e. much higher frequency), $13 \mathrm{~cm}$ and $116 \mathrm{~cm}$, in their observations, and that, observed RMS gradient varies depending on the observation frequency as seen in figure 9.75 of Heiken et al. (1991); higher frequency gives larger RMS gradient. With higher frequency (shorter wavelength) one observes smaller structures of lunar surface such as numerous small craters, rocks, pebbles, sand grains, and regolith particles. With lower frequency (longer wavelength) one observes more global structures, or averaged features of lunar surface.

Yet, another possibility is that the discrepancy may be attributed to that those ALSE observation quoted in the present paper was, possibly by chance, carried out in a particularly smooth surface area.

The answer will be given by LRS observation data.

\section{Conclusion}

Computer simulations of LRS observation have been conducted by utilizing newly developed the KiSS code in order to understand the reflection and refraction nature of HF waves and to confirm that the geologic structure of lunar subsurface can be investigated from orbit by means of an HF radar.

From the results of the simulations, we have found that the power flux of both surface nadir echo and subsurface nadir echo vary little if roughness of either/both surface or/and subsurface boundary interface varies. However, the power flux of surface off-nadir backscattering echo changes following a power law of $\left(k \sigma_{0}\right)^{2}$ where $k$ is the wavenumber of LRS pulse and $\sigma_{0}$ is the RMS height of the surface feature. Thus slight roughness of the surface causes significant increase of the intensity of surface off-nadir backscattering echo, which easily masks weak subsurface echo. These observations have been understood qualitatively by geometrical optics approximation and quantitatively by examining the Stratton's integral formula analytically.

Computer simulations have revealed that subsurface echoes can be observed even if it is completely masked by 
surface off-nadir backscattering echo, thus, indistinguishable in an A-scope display. To distinguish those subsurface echoes from intense surface off-nadir backscattering echoes, data stacking technique has been proved to be effective in reducing the intensity of surface off-nadir echoes due to their random nature.

Lastly, simulation results have been cross checked with ALSE results, and the RMS surface gradient of a lunar mare region has been estimated to be about $1.3^{\circ}$.

Although the present simulation study has been carried out for a case of specific model parameters such as that the depth of subsurface boundary interface is $1,000 \mathrm{~m}$, the nature of both surface and subsurface reflection and that of data stacking method are independent of those model parameters. Therefore the results of the present study confirm that LRS will be a powerful tool to explore lunar subsurface structure.

Acknowledgments. The present research was carried out as a basic development study of the Lunar Radar Sounder experiment by SELENE project. The KiSS code has been developed as a collaboration with Super Computing System, Information Synergy Center, Tohoku University. The authors would like to thank them for their consultation and advice on development of the KiSS code. And the authors appreciate Dr. T. Okada and the anonymous referee for their careful reading of the manuscript and their advice.

\section{References}

Beckmann, P. and A. Spizzichino, The Scattering of Electromagnetic Waves from Rough Surfaces, Reprinted 1987 by Artech House Inc., Norwood, Massachussetts, USA, 1963.

Cooper, B. L., Applications of electromagnetic radiation for exploration of lunar regolith for potential resources, $\mathrm{PhD}$ thesis, The university of Texas at Dallas, 1992.

Cooper, B. L., J. L. Carter, and C. A. Sapp, New evidence for graben origin of Oceanus Procellarum from lunar sounder optical imagery, J. Geophys. Res., 99(E2), 3799-3812, 1994.

Heiken, G., D. Vaniman, and F. M. French, (ed.), Lunar Sourcebook, Cambridge University Press, Cambridge, 1991.

Kobayashi, T., H. Oya, and T. Ono, B-scan analysis of subsurface radar sounding of lunar highland region, Earth Planets Space, 54, this issue 983-991, 2002.

Ogilvy, J. A., Theory of Wave Scattering from Random Rough Surfaces, Institute of Physics Publishing, Philadelphia, USA, 1992.
Olhoeft, G. R. and D. W. Strangway, Dielectric properties of the first 100 meters of the moon, Earth Planet. Sci. Lett., 24, 394-404, 1975.

Ono, T. and H. Oya, Lunar Radar Sounder (LRS) experiment on-board the SELENE spacecraft, Earth Planets Space, 52, 629-637, 2000.

Ono, T., H. Oya, A. Morioka, A. Kumamoto, K. Kobayashi, T. Obara, and T. Nakagawa, Plasma waves and sounder (PWS) experiment onboard the Planet-B Mars orbiter, Earth Planets Space, 50, 213-221, 1998.

Oya, H., T. Ono, and T. Kamada, Stimulation of plasma waves in the magnetosphere using satellite Jikiken (EXOS-B) Part I. observation of plasma resonances, J. Geomag. Geoelectr., 33, 3-25, 1981.

Oya, H., A. Morioka, and T. Obara, Leaked AKR and terrestrial hectometric radiations discovered by the plasma wave and plasma sounder experiments on board the Ohzora (EXOS-C) satellite - instrumentation and observation results of plasma wave phenomena, J. Geomag. Geoelectr., 37, 247-262, 1985.

Oya, H., A. Morioka, K. Kobayashi, M. Iizima, T. Ono, H. Miyaoka, T. Okada, and T. Obara, Plasma wave observation and sounder experiments (PWS) using the Akebono (EXOS-D) satellite-instrumentation and initial results including discovery of the high altitude equatorial plasma turbulence, J. Geomag. Geoelectr., 42, 411-442, 1990.

Peeples, W. J., W. R. Sill, T. W. May, S. H. Ward, R. J. Phillips, R. J. Jordan, E. A. Abbott, and T. J. Killpack, Orbital radar evidence for lunar subsurface layering in maria Serenitatis and Crisium, J. Geophy. Res., 83(B7), 3459-3468, 1978

Phillips, R. J., G. F. Adams, Jr., W. E. Brown, R. E. Eggleton, P. Jackson, R. Jordan, W. I. Linlor, W. J. Peeples, L. J. Porcello, J. Ryu, G. Schaber, W. R. Sill, T. W. Thompson, S. H. Ward, and J. S. Zelenka, Apollo Lunar Sounder Experiment, Chap. 22, Apollo 17 Preliminary Science Report, NASA SP-330, 1973.

Simpson, R. A., Surface roughness estimation at three points on the lunar surface using 23-cm monostatic radar, J. Geophys. Res., 81, 4407-4416, 1976.

Simpson, R. A. and G. L. Tyler, Radar scattering laws for the lunar surface, IEEE Transaction of Antennas and Propagation, AP-30, 438-449, 1982.

Stratton, J. A., Electromagnetic Theory, McGrqw-Hill, New York, 1941.

Thomas, T. R., Rough Surface, second edition, Imperial Collage Press, London, 1999.

Tyler, G. L. and H. T. Howard, Dual frequency bistatic radar investigation of the moon with Apollo 14 and 15, J. Geophys. Res., 78, 4852-4874, 1973.

Yamaji, A., S. Sasaki, Y. Yamaguchi, T. Ono, J. Haruyama, and T. Okada, Lunar tectonics and its implications for the origin and evolution of the moon, Mem. Geol. Soc. Japan, 50, 218-226, 1998.

T. Kobayashi (e-mail: tak@stpp1.geophys.tohoku.ac.jp), H. Oya, and T. Ono 